\title{
Dissection of the internal carotid artery causing Horner syndrome and palsy of cranial nerve XII
}

\author{
Neema Kasravi MD, Andrew Leung MD, Ian Silver MD MSc, Jorge G. Burneo MD MSPH
}

Previously published at www.cmaj.ca

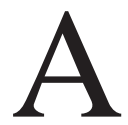

61-year-old right-handed man presented with a twoday history of a new headache that had developed gradually several hours after he dived into a lake. The headache, which encompassed the entire left side of the head, had a maximal intensity of 10 out of 10 and was described as dull and constant with mild photophobia. The patient also reported blurry vision in the left eye. In addition, his speech was slurred, and he had difficulty swallowing.

There were no carotid or vertebral artery bruits evident on physical examination. Cranial nerve examination demonstrated 2.5-mm ptosis of the left eye; in addition, the diameter

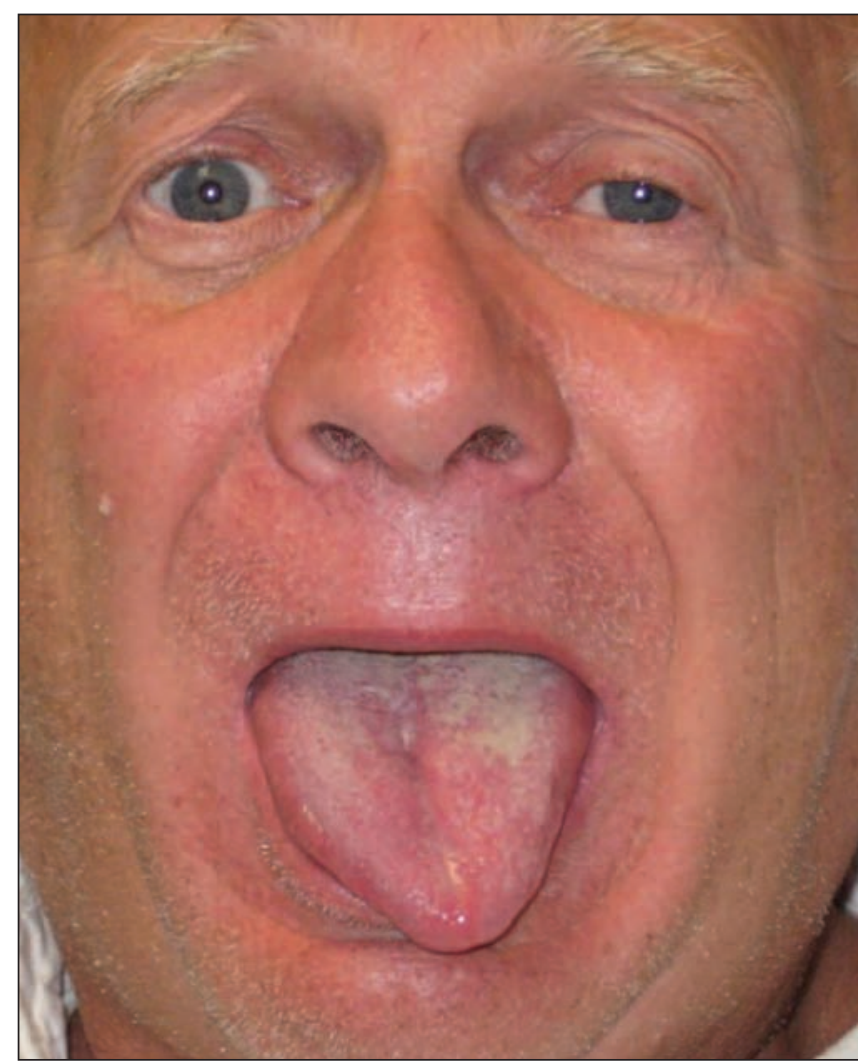

Figure 1: Photograph of a 61-year-old man with intense leftsided headache, showing leftward deviation of the tongue and ptosis and miosis of the left eye.

\section{Key points}

- Dissection of the carotid artery should be considered in cases of new unilateral headache in patients with a history of trauma or with accompanying Horner syndrome or cranial nerve palsy.

- Computed tomography angiography and magnetic resonance angiography are sensitive diagnostic imaging modalities for carotid artery dissection and should be used before digital subtraction (conventional) angiography.

- Magnetic resonance angiography has a higher sensitivity after several days, as the hemoglobin in the mural hematoma of the dissection will have been converted to methemoglobin, which is hyperintense on $\mathrm{T}_{1}$-weighted sequences.

- Carotid artery dissection may be associated with significant morbidity because of cerebral ischemia and requires prompt treatment with antiplatelet or anticoagulation therapy.

of the left pupil was $1 \mathrm{~mm}$ smaller than that of the right in dim light (Figure 1). Both pupils were reactive, and there was no relative afferent pupillary defect. The tongue deviated to the left when protruded; there were no fasciculations (Figure 1). The results of the remainder of the cranial nerve examination were normal, including normal gag response, symmetric elevation of the palate and full power in the sternocleidomastoid and trapezius muscles. The results of motor, sensory, cerebellar and gait testing were unremarkable. Ophthalmoscopy and visual-field testing during a neuro-ophthalmologic consultation did not reveal any additional abnormalities.

At this point, the main diagnostic considerations included a cervical mass lesion within the carotid sheath, such as dissection of the carotid artery, which could cause a mass effect on the adjacent ascending sympathetic plexus or on cranial nerve XII at the base of the skull, beyond the point of emergence from the hypoglossal foramen. Such a mass would cause ipsilateral Horner syndrome (see Box 1) and deviation of the tongue. Alternatively, a left-sided lesion of the brainstem

From the Department of Clinical Neurological Sciences (Kasravi, Burneo) and the Department of Medical Imaging (Leung, Silver), London Health Sciences Centre, The University of Western Ontario, London, Ont.

CMAJ 2010. DOI:10.1503/cmaj.091261 


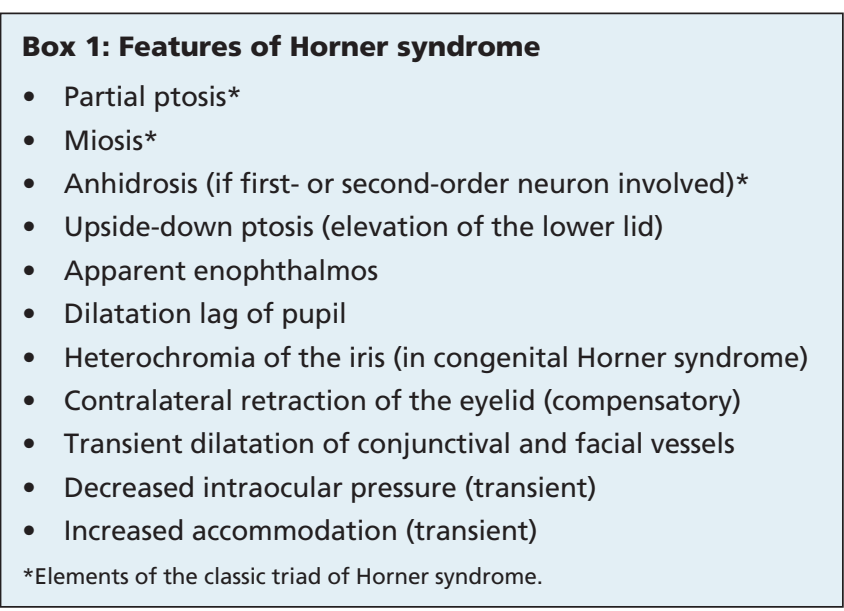

could affect the descending sympathetic tract and the nucleus and fascicle of cranial nerve XII where they lie close to one another in the medulla. Although medullary ischemic stroke and hemorrhage were entertained as possible diagnoses, they were considered less likely than a mass localized within the carotid sheath, given the distribution of the headache and the absence of associated dysfunction of the brainstem. The expected deficits with such a brainstem lesion would include signs and symptoms of medial medullary syndrome (such as ipsilateral deviation of the tongue) and lateral medullary syndrome (such as ipsilateral Horner syndrome) (Box 2).

Computed tomography (CT) and CT angiography of the neck demonstrated subtle, concentric mural soft-tissue thickening of the left internal carotid artery immediately proximal

\section{Box 2: Signs of medial and lateral medullary syndromes}

Medial medullary syndrome (Dejerine syndrome)

- Ipsilateral hypoglossal palsy (hemiatrophy and ipsilateral deviation of tongue)

- Contralateral impairment of fine touch, vibration and proprioception

- Contralateral hemiparesis or hemiplegia

- Ipsilateral palatal and pharyngeal myorhythmia

- Nystagmus

Lateral medullary syndrome (Wallenberg syndrome)

- Horner syndrome

- Ipsilateral ataxia

- Contralateral impairment of sensation of pain and temperature of the body

- Ipsilateral impairment of sensation of pain and temperature of the face

- Nystagmus

- Ipsilateral loss of hearing

- Hoarse voice and/or dysphagia (ipsilateral paresis of palate, larynx or pharynx)

- Cardiac arrhythmia

- Loss of taste

- Hiccups

- Palatal and pharyngeal myorhythmia to the petrous portion of the vessel (Figure 2A). Magnetic resonance imaging (MRI) with magnetic resonance angiography, including a $\mathrm{T}_{1}$-weighted fat-saturation sequence, performed on day three of symptoms, showed no definite evidence of dissection, infarct or brainstem lesion (Figure 2B). Cerebral digital subtraction angiography did not demonstrate any irregularity of the carotid artery or narrowing that would indicate dissection (Figure 3). Repeat MRI on day eight demonstrated high $\mathrm{T}_{1}$-weighted signal within the wall of the distal extracranial and petrous segments of the left internal carotid artery (Figure $2 \mathrm{C})$. This finding was diagnostic of subacute dissection of the internal carotid artery and confirmed the initial CT findings.

The patient was started on acetylsalicylic acid (ASA) 81 mg daily. At follow-up six weeks after the initial presentation, there was significant improvement in all symptoms. Followup MRI at six months revealed resolution of the mural hematoma in the left internal carotid artery (Figure 2D). By that time, the patient's headache, miosis, ptosis, dysphagia and dysarthria had almost completely resolved.

\section{Discussion}

Dissection of the carotid artery occurs when a tear forms within the inner wall of an artery. Blood enters the tunica media of the vessel and forms an intramural hematoma along

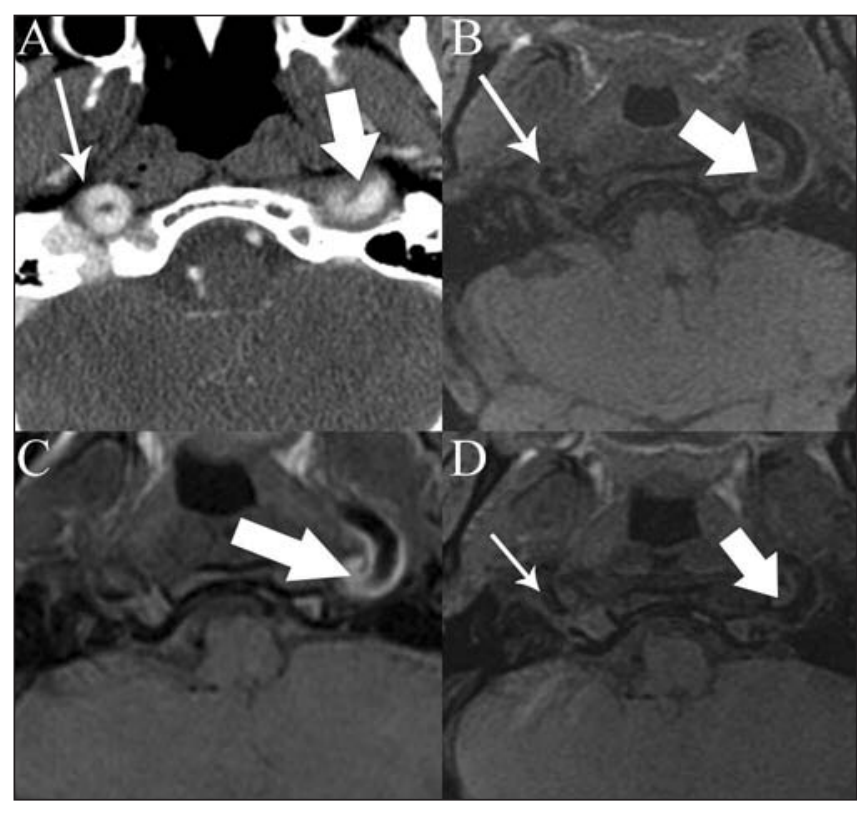

Figure 2: A: Transverse computed tomography angiography of the neck demonstrates subtle mural soft-tissue thickening of the left internal carotid artery on day two of symptoms (large arrow). B: Transverse $\mathrm{T}_{1}$-weighted magnetic resonance imaging (MRI) with fat saturation on day three of symptoms is consistent with a normal left internal carotid artery (large arrow). C: Transverse $\mathrm{T}_{1}-$ weighted MRI with fat saturation on day eight of symptoms demonstrates new mural hyperintensity and thickening of the left internal carotid artery. D: Transverse $\mathrm{T}_{1}$-weighted MRI with fat saturation at six months after presentation shows resolution of the soft-tissue hyperintensity and thickening of the left internal carotid artery. The tortuous but otherwise normal right internal carotid artery is indicated by a small arrow in some of the images. 
the plane of the vessel wall (Figure 4). This may cause the vessel wall to bulge toward the lumen, leading to stenosis, or it may cause outward pseudoaneurysmal bulging of the vessel wall. The incidence of dissection of the internal carotid artery is $2.5-3.0$ per $100000 .^{1}$

Dissection may occur secondary to trauma or spontaneously, although minor trauma such as coughing probably goes unrecognized as a cause. In one study of 1313 people with blunt trauma, patients meeting specific clinical criteria were screened with CT angiography. ${ }^{2}$ Fifty-three percent of the study population had sustained their injuries in motor vehicle crashes. About $1 \%$ of the study population (19 subjects) had injury to the cervical vessels detected with CT angiography with or without confirmatory digital subtraction angiography. ${ }^{2}$ Five of these patients had injuries to the extracranial internal carotid artery, 13 had injuries to the

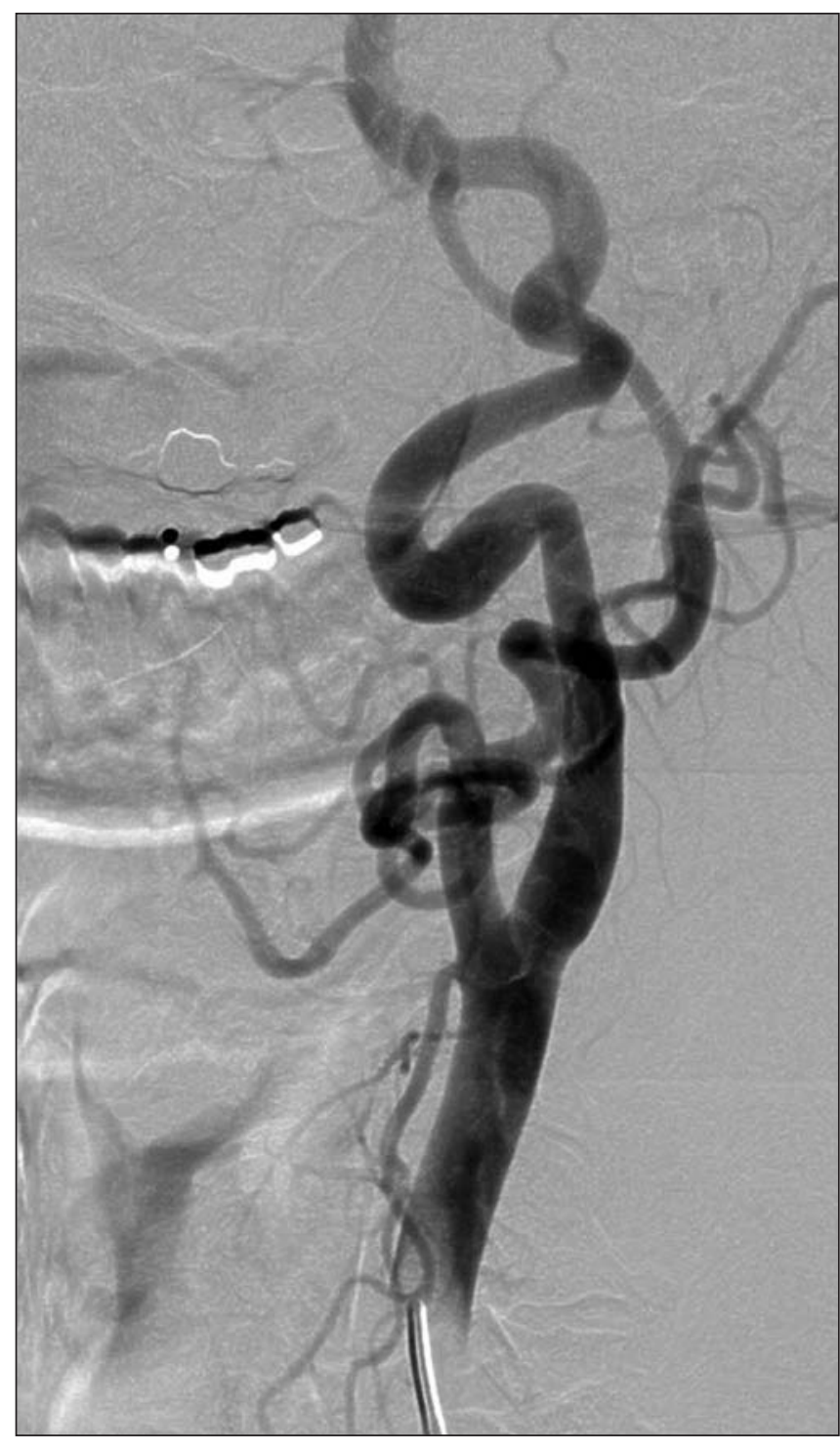

Figure 3: Digital subtraction angiogram after injection of contrast medium into the left common carotid artery on day six of symptoms shows some tortuosity of the vessel but no convincing mural irregularity or luminal narrowing. extracranial vertebral arteries, and one had injury of the common carotid artery.

Heritable connective tissue disorders associated with spontaneous dissection of the internal carotid artery include Ehlers-Danlos syndrome type IV, Marfan syndrome, autosomal dominant polycystic kidney disease and osteogenesis imperfecta type I, although these disorders are rarely detected

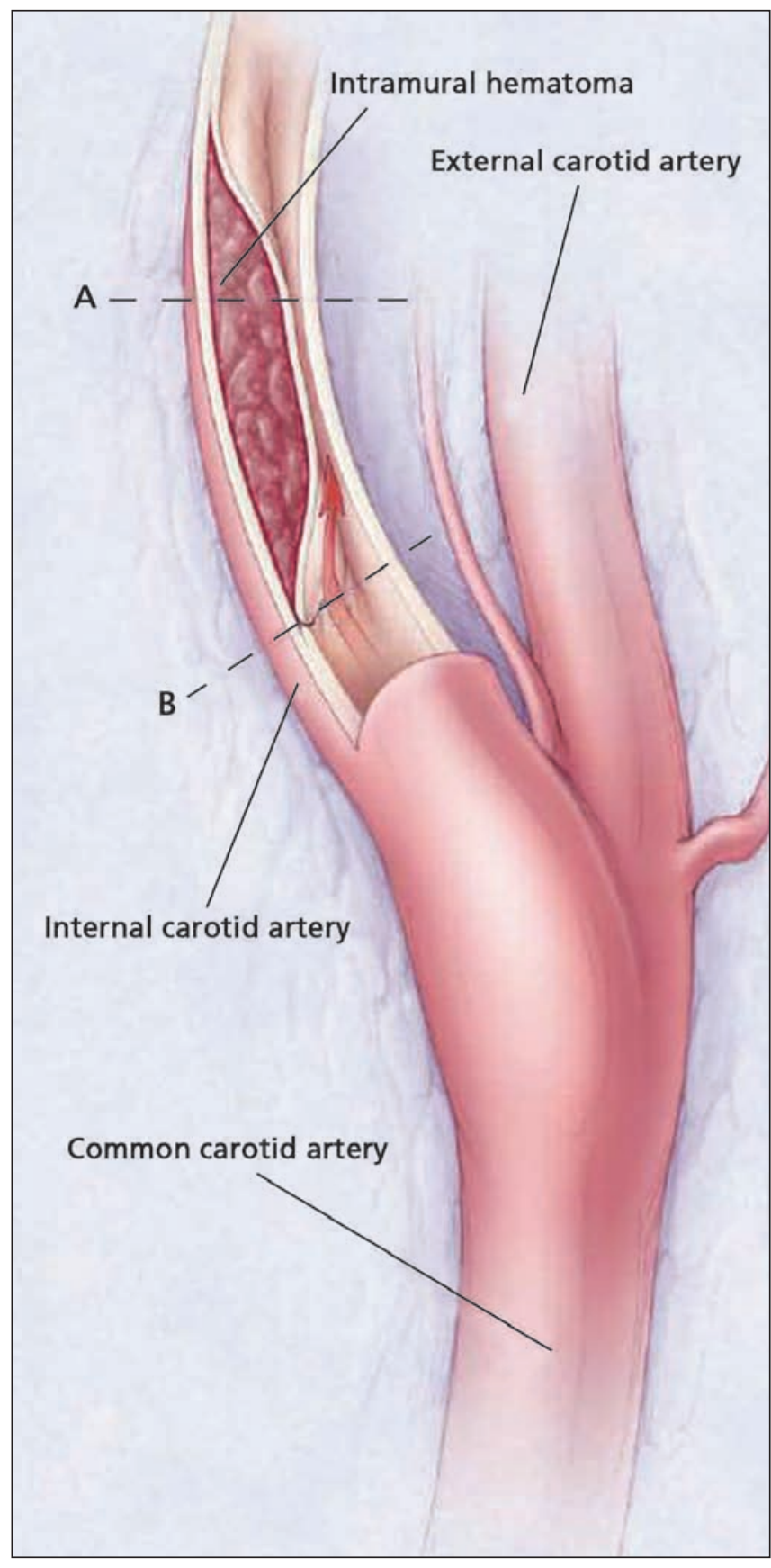

Figure 4: Dissection of the internal carotid artery (site B) causes a mural hematoma to form in the tunica media of the vessel wall (site A). The mural hematoma is causing stenosis of the vessel's lumen. Reproduced, with permission, from Schievink WI. ${ }^{1}$ Spontaneous dissection of the carotid and vertebral arteries. N Engl J Med 2001;344: 898-906. Copyright 2001 Massachusetts Medical Society. All rights reserved. 


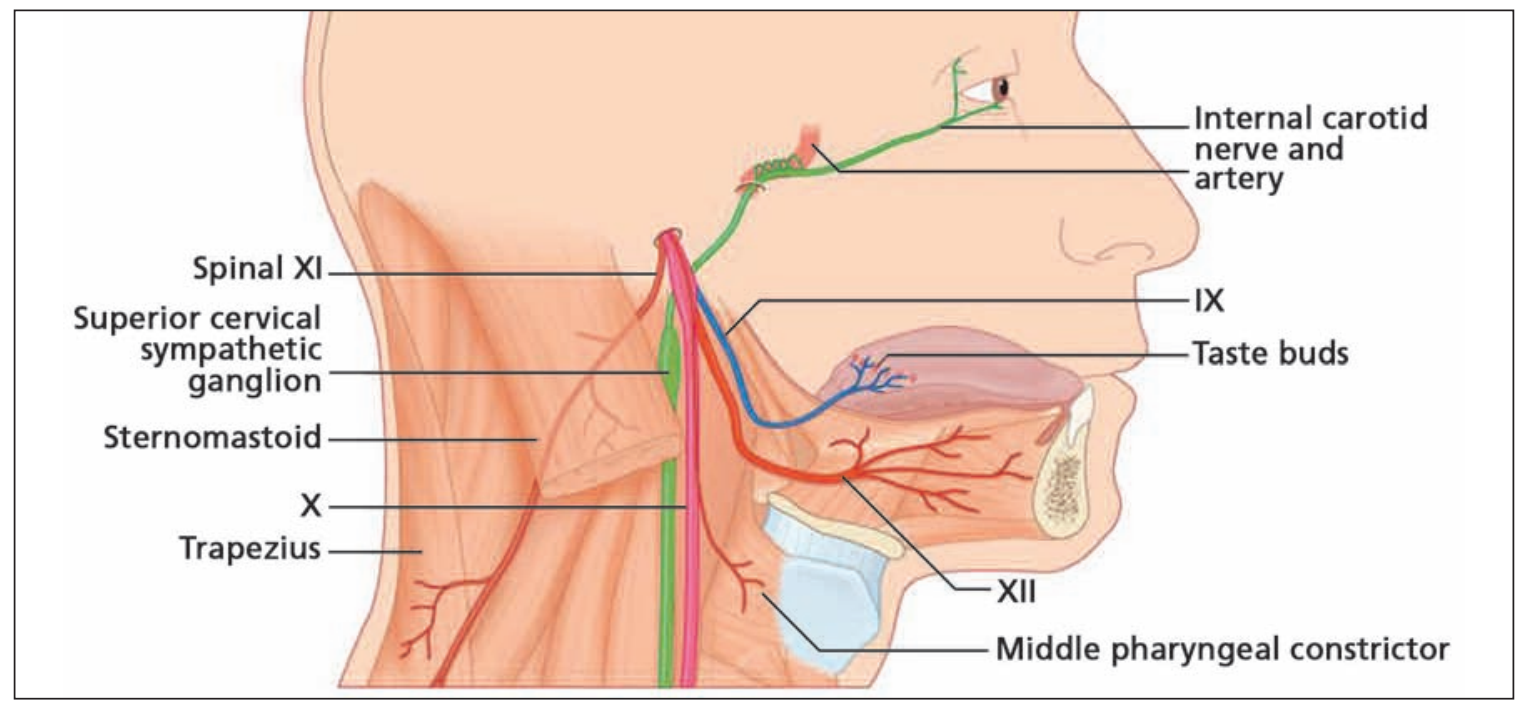

Figure 5: The lowest four cranial nerves are shown emerging from the jugular and hypoglossal foramina, where they join the sympathetic plexus within the carotid sheath. Here, these structures are vulnerable to the compressive effects of a mural hematoma resulting from a carotid dissection. Reproduced, with permission, from FitzGerald MJT, Gruener G, Mtui E. ${ }^{6}$ Clinical Neuroanatomy and Neuroscience, 5th ed. Figure 18.2. Copyright 2007 Elsevier.

in the setting of cervical artery dissection. ${ }^{1}$ Dissection of the carotid and vertebral arteries is an important cause of ischemic stroke in young and middle-aged patients, accounting for $10 \%-25 \%$ of strokes in this age group. ${ }^{1.3}$

Dissection of the extracranial portion of the internal carotid artery is much more frequent than intracranial dissection, representing more than $90 \%$ of all dissections in the carotid system. Extracranial dissection of the internal carotid artery usually starts $2 \mathrm{~cm}$ distal to the carotid bulb, near the level of the second or third cervical vertebra. ${ }^{4}$

\section{Clinical presentation}

The most common symptoms of dissection of the carotid artery are ipsilateral cervical pain, which occurs in one-quarter of patients, and headache, which occurs in two-thirds of patients. ${ }^{1}$ Horner syndrome, characterized by ptosis and miosis, is caused by compression of the ascending sympathetic supply within the carotid sheath ${ }^{1}$ and occurs in fewer than half of affected patients (Box 1). Facial sweating is usually not affected, as the sympathetic supply to the sweat glands ascends along the external carotid plexus. Patients may also present with symptoms of transient ischemic attack (50\%-95\% of cases $\left.{ }^{1}\right)$ within hours to days after the dissection occurs. Less commonly, patients may have an audible bruit or cranial nerve palsies. ${ }^{5}$ Extracranial dissection of the internal carotid artery causes cranial nerve palsy in $12 \%$ of cases. ${ }^{5}$ Cranial nerve XII is the most commonly affected, in roughly $5 \%$ of patients. There is variable involvement of the other lower cranial nerves, caused by local compressive effects in the carotid sheath or by compromise of feeder vessels to these nerves ${ }^{5}$ (Figure 5).

\section{Diagnosis}

A variety of imaging techniques are available for the diagnosis of carotid artery dissection, but all have limitations. Historically, digital subtraction angiography has been considered the gold standard. ${ }^{7}$ However, this method is not a true gold standard, because it will not reveal a dissection that lacks luminal irregularity or narrowing, as in the case presented here. Because of the associated costs and invasiveness of digital subtraction angiography, MRI with magnetic resonance angiography and CT with CT angiography are used more routinely. MRI allows for visualization of infarcts beyond the dissection through diffusion-weighted imaging sequencing, and $\mathrm{T}_{1}$-weighted imaging can demonstrate a mural hematoma as a hyperintense crescent sign. The drawbacks of MRI include high cost, limited availability and issues related to patient tolerance and contraindications. Additionally, in the first few days after dissection occurs, the mural hematoma consists primarily of deoxyhemoglobin, which does not have hyperintense $\mathrm{T}_{1}$-weighted signal on MRI and can easily be missed. ${ }^{8}$

CT is much faster and more readily available. However, this imaging modality exposes the patient to ionizing radiation, and mural hematoma may be mistaken for noncalcified atherosclerotic plaque. Carotid duplex ultrasonography may also demonstrate the features of a carotid dissection, but it lacks the sensitivity of the other modalities described.

Decisions about the imaging modality must be made on a case-by-case basis, bearing in mind the strengths and weaknesses of each method. The evidence suggests that in the first few days following the onset of symptoms, CT with CT angiography is very sensitive ${ }^{9}$ for detecting cervical artery dissection and may be favoured over magnetic resonance angiography and digital subtraction angiography. ${ }^{7,10}$ Several days after the onset of symptoms, MRI or magnetic resonance angiography would have higher sensitivity and specificity than in the acute period, as the hemoglobin in the hematoma will have been converted to more readily identifiable methemoglobin. Digital subtraction angiography should be performed as a last resort and only if it is clinically indicated. 


\section{Treatment}

Patients are treated empirically with either antiplatelet or anticoagulation therapy to prevent formation of a thrombus at the site of dissection and subsequent embolization. However, no studies have shown a benefit of one treatment over the other. ${ }^{11,12}$ With anticoagulation, there remains a risk of extension of the dissection and development of intramural hematoma and other adverse events caused by bleeding. In general, anticoagulation is avoided for dissections in the intracranial segment of the artery, because of the risk of subarachnoid hemorrhage. Some clinicians maintain patients on lifelong low-dose ASA, although again, there is no supporting evidence for this practice.

Currently, endovascular and surgical repair of the internal carotid artery should be considered only for patients with deteriorating or fluctuating neurologic symptoms resulting from thromboemboli or cerebral hypoperfusion refractory to conservative medical management and only if there is good collateral circulation. Unfortunately, published case series for these interventions are small, and information about longterm outcomes is lacking. Intervention is also indicated in the setting of chronic dissection, where there is persistent, highgrade stenosis of the vessel or a large persistent pseudoaneurysm. Although minimally invasive techniques are now the preferred treatment modality in these cases, the long-term efficacy and durability of a stent in cases of arterial dissection remain to be determined..$^{13,14}$

Patients are usually followed regularly for several months to a year to ensure clinical resolution of symptoms. Serial imaging may also be used to monitor resolution of the mural hematoma and repair of the vessel wall.

This article has been peer reviewed.

Competing interests: Dr. Burneo received an honorarium from UCB Pharma Canada Inc. in December 2008 to attend a board advisory meeting in Seattle, USA. None declared for Dr. Kasravi, Dr. Leung and Dr. Silver.

Contributors: Dr. Kasravi was the primary manuscript author. Drs. Burneo, Leung and Silver were involved in the critical revision of the manuscript. All authors approved of the final manuscript submitted for publication.

Acknowledgement: The authors acknowledge the clinical contribution of Dr. David Nicolle in the neuro-ophthalmological evaluation of this case.

\section{REFERENCES}

1. Schievink WI. Spontaneous dissection of the carotid and vertebral arteries. $N$ Engl J Med 2001;344:898-906

2. Schneidereit NP, Simons R, Nicolaou S, et al. Utility of screening for blunt vascular neck injuries with computed tomographic angiography. J Trauma 2006;60:209-15.

3. Leys D, Bandu L, Henon H, et al. Clinical outcome in 287 consecutive young adults (15-45 years) with ischemic stroke. Neurology 2002;59:26-33.

4. Guillon B, Lévy C, Bousser MG. Internal carotid artery dissection: an update. $J$ Neurol Sci 1998;153:146-58.

5. Mokri B, Silbert PL, Schievink WI, et al. Cranial nerve palsy in spontaneous dissection of the extracranial internal carotid artery. Neurology 1996;46:356-9.

6. FitzGerald MJT, Gruener G, Mtui E. Clinical neuroanatomy and neuroscience. 5th ed. Elsevier; 2007.

7. Vertinsky AT, Schwartz NE, Fischbein NJ, et al. Comparison of multidetector CT angiography and MR imaging of cervical artery dissection. AJNR Am J Neuroradiol 2008;29:1753-60.

8. Leclerc X, Lucas C, Godefroy $\mathrm{O}$, et al. Preliminary experience using contrastenhanced MR angiography to assess vertebral artery structure for the follow-up of suspected dissection. AJNR Am J Neuroradiol 1999;20:1482-90.

9. Chen CJ, Tseng YC, Lee TH, et al. Multisection CT angiography compared with catheter angiography in diagnosing vertebral artery dissection. AJNR Am J Neuroradiol 2004;25:769-74

10. Lum C, Chakraborty S, Schlossmacher M, et al. Vertebral artery dissection with a normal-appearing lumen at multisection $\mathrm{CT}$ angiography: the importance of identifying wall hematoma. AJNR Am J Neuroradiol 2009;30:787-92.

11. Lyrer P, Engelter S. Antithrombotic drugs for carotid artery dissection. Cochrane Database Syst Rev 2003;(2):CD000255.

12. Georgiadis D, Arnold M, von Buedingen HC, et al. Aspirin vs anticoagulation in carotid artery dissection: a study of 298 patients. Neurology 2009;72:1810-5.

13. Müller BT, Luther B, Hort W, et al. Surgical treatment of 50 carotid dissections: indications and results. J Vasc Surg 2000;31:980-8.

14. Joo JY, Ahn JY, Chung YS, et al. Treatment of intra- and extracranial arterial dissections using stents and embolization. Cardiovasc Intervent Radiol 2005;28:595-602.

Correspondence to: Dr. Neema Kasravi, University Hospital, Department of Clinical Neurologic Sciences, London Health Sciences Centre, 339 Windermere Rd., London ON N6A 5A5; nkasravi@uwo.ca

The section Cases presents brief case reports that convey clear, practical lessons. Preference is given to common presentations of important rare conditions, and important unusual presentations of common problems. Articles start with a case presentation (500 words maximum), and a discussion of the underlying condition follows (1000 words maximum). Generally, up to five references are permitted and visual elements (e.g., tables of the differential diagnosis, clinical features or diagnostic approach) are encouraged. Written consent from patients for publication of their story is a necessity and should accompany submissions. See information for authors at www.cmaj.ca. 